\title{
ANÁLISE DA COMERCIALIZAÇÃO DE MEDICAMENTOS E PRODUTOS PARA EMAGRECER EM UMA DROGARIA NO MUNICÍPIO DE CERES-GO
}

Suzana Bruni Tavares ${ }^{1}$; Letícia Jaqueline de Oliveira Ângelo; Maria Juíva Marques de Faria Souza ${ }^{2}$

${ }^{1}$ Discentes do Curso de Farmácia, Faculdade Evangélica de Ceres, Ceres-GO, Brasil.

${ }^{2}$ Docente Mestra, Faculdade Evangélica de Ceres, Ceres-GO, Brasil

(juivamaria@hotmail.com).

Recebido em: 08/04/2017 - Aprovado em: 10/06/2017 - Publicado em: 20/06/2017 DOI: 10.18677/EnciBio_2017A119

\begin{abstract}
RESUMO
Com intuito de obter um corpo ideal e de forma rápida, uma das classes medicamentosas mais utilizadas pelas mulheres são os anorexígenos. Outros produtos que destacam é o orlistat, a spirulina, os fitoterápicos, as drogas e derivados vegetais. $O$ objeto do estudo foi analisar a comercialização de medicamentos e produtos para emagrecer em uma drogaria no município de CeresGO. Tratou-se de uma pesquisa de campo observacional de análise quantitativa descritiva de corte transversal. Verificou o gênero que mais comprou medicamentos para emagrecer, se produto farmacêutico apresentava prescrição médica ou era isento de prescrição, o medicamento mais dispensado para tal finalidade, a especialidade médica que mais emitiu receituários e se os farmacêuticos orientaram os pacientes frente ao tratamento medicamentoso. O gênero que mais comprou medicamento para emagrecer foi o feminino com $75 \%(24 / 32)$ dos pacientes, predominou a dispensação de MIP's com 46,88\% (15/32), a sibutramina foi o medicamento mais comercializado com $71,42 \%(10 / 14)$, o óleo de coco foi 0 derivado vegetal mais vendido durante o estudo com $40 \%(04 / 10)$, em relação a outras classes de produtos farmacêuticos destacou o orlistat com 87,50\% (07/08), com $66,67 \%(08 / 12)$ o endocrinologista foi o que mais indicava tais medicamentos e com 68,75\% (22/32) de pacientes não receberam orientação farmacêutica frente ao tratamento medicamentoso. Conclusão: As mulheres se preocupam mais com a estética do corpo e se automedicam com maior frequência. A sibutramina foi o medicamento mais comercializado, desta forma, é importante o papel do farmacêutico em informar, alertar os riscos e benefícios dos medicamentos.
\end{abstract}

PALAVRAS-CHAVE: Anorexígenos. Atenção farmacêutica. Automedicação. 


\title{
EVALUATION OF THE MARKETING OF MEDICINAL PRODUCTS AND MEDICAL PRODUCTS IN A DRUG IN THE MUNICIPALITY OF CERES- GO
}

\begin{abstract}
Among the drug classes most used in the search for the ideal body and quickly are the anorectics. Other products that also stand out are orlistat, spirulina, phytotherapics, drugs and vegetable derivatives. The object of the study was evaluate the commercialization of drugs and products for weight loss, in a drugstore in the municipality of Ceres-GO. This was an observational field study of descriptive quantitative cross-sectional analysis, it was verified the gender that bought the most drugs to lose weight, whether the pharmaceutical product had a medical prescription or was exempt from prescription, the most widely used medicinal product for this purpose, the medical specialty that issued the most prescription drugs and whether pharmacists advised patients against drug treatment. The gender that most bought drugs to lose weight was womem, with $75 \%(24 / 32)$ of the patients. The dispensation of MIPs was predominant, with $46.88 \%$ (15/32). Sibutramine was the most marketed drug, with $71.42 \%(10 / 14)$. Coconut oil was the most sold vegetable derivative during the study, with $40 \%(10 / 10)$. In relation to other classes of pharmaceutical products orlistat with $87.50 \%(07 / 08)$ stood out, with $66.67 \%(08 / 12)$ the endocrinologist was the professional who most indicated such medications and wihy $68.75 \%(22 / 32)$ of the patients did not receive pharmaceutical orientation against drug treatment. Women are more concerned with body aesthetics and self-medicate more frequently. Sibutramine was the most marketed drug. Thus, the role of the pharmacist in informing and alerting patients about the risks and benefits of medicines is important. KEYWORDS: Anorexigenics. Pharmaceutical attention. Self-medication.
\end{abstract}

\section{INTRODUÇÃO}

A procura incessante pela beleza, a preocupação com a própria imagem, são situações muito comuns nos dias atuais (MELO \& OLIVEIRA, 2011), sendo também um fator que contribui com o consumismo exagerado. Essa busca pela manutenção da beleza, é considerada uma preocupação que percorre pelo mundo feminino por vários séculos (DUTRA et al., 2015), pois a sociedade cria estereótipos da imagem corporal, definindo a estética do corpo com os padrões de beleza (SILVA et al., 2012).

Desta forma, muita das vezes, a sociedade busca no medicamento uma solução imediatista e milagrosa para seus insucessos, frustações e patologias, como por exemplo, a obesidade e insatisfação do corpo (MARQUES, 2008). Ressalta-se que uma consequência negativa disso é a automedicação, ou seja, um autoconsumo de medicamento sem o acompanhamento de um profissional da saúde, com o intuito de aliviar sintomas percebidos pelo paciente (ROSSE et al., 2011).

Embora algumas mulheres tenham o conhecimento do risco da automedicação e do seu uso abusivo, arriscam-se no uso irracional de medicamentos para ter um corpo lindo, magro e aumentar a autoestima. Muitas das vezes, mesmo com o profissional da saúde contraindicando o uso de determinado medicamento, a maioria das mulheres insistem no uso destes e não buscam outros métodos, como a prática de exercícios físicos e a reeducação alimentar (MELO \& OLIVEIRA, 2011) pois, acreditam que a prática do uso de medicamentos proporciona tratamento mais rápido e eficiente (CARVALHO \& MACIEIRA, 2012). 
Consequências graves da automedicação são as intoxicações, reações alérgicas e dependências e em alguns casos, a pessoa pode chegar ao óbito (LAGARES, 2015). Segundo Abifarma cerca de 20 mil pessoas morrem anualmente vítimas da automedicação. Isso é devido a vida moderna e a dificuldade de acesso aos médicos e consultas, tornando a automedicação uma prática perigosa (ABIFARMA, 2015).

Com intuito de emagrecer mais rápido e obter um corpo ideal, uma das classes medicamentosas mais utilizadas pelas mulheres com idade média entre $21 \mathrm{e}$ 30 anos são os anorexígenos (SILVA et al., 2012). Dentre estes destacavam no Brasil a anfepramona, o femproporex, o mazindol e a sibutramina, os quais agem sobre o sistema nervoso central liberando substâncias que transmitem a sensação de ausência de fome (ABESO, 2010). De acordo com os dados citados pelo Dirceu Barbano ex-Diretor presidente da Agência Nacional de Vigilância Sanitária, o Brasil é responsável por cerca de $50 \%$ da sibutramina vendida em todo o mundo, e ocupa também o terceiro lugar no ranking dos países que mais consomem derivados anfetamínicos (BRASIL, 2011 b). Destaca-se que a prescrição, dispensação e o aviamento de medicamentos que contenham essas substâncias deverão ser realizados por meio da Notificação de Receita "B2" (BRASIL, 2011 a).

Outros medicamentos que destacam no mercado com a finalidade de contribuir com a perda de peso é o orlistate e a spirulina. O orlistate tem uma ação intestinal, inibindo as lípases pancreáticas, que reduz 30\% das gorduras que são ingeridas, sendo eliminadas pela excreção fecal. Já a spirulina ocorre uma ação supressora do apetite pois, quando é ingerida com o estômago vazio, produz sensação de saciedade (PRADO et al., 2010).

Há também os medicamentos fitoterápicos, drogas vegetais e derivados vegetais muito utilizados pela população com a finalidade de emagrecer. Dentre estas destacam-se a Camelia sinensis (chá verde), Cynara scolymus (alcachofra), Baccharis trimera (carqueja), Equisetum giganteum (cavalinha), Hibiscus rosasinensis (hibisco), óleo de coco, óleo de cártamo e entre outros (COSTA et al., 2015; WEISHEIMER et al., 2015; MARTINS \& GARLET, 2016). Dentre estes medicamentos usados há também a associação com outras classes medicamentosas como os laxantes, antidepressivos e diuréticos (SILVA et al., 2012).

Desta forma, é importante o papel do farmacêutico na assistência e atenção farmacêutica, que tem como objetivo informar e alertar os riscos e benefícios dos medicamentos anorexígenos, orientar quanto às possíveis interações medicamentosas e problemas relacionados ao uso incorreto (posologia) dos medicamentos pois, através da atenção farmacêutica o farmacêutico pode realizar essas orientações e também propor métodos não farmacológicos, como a prática de exercícios físicos e orientação na reeducação alimentar dos pacientes. Além disso, é importante destacar que deve ter uma interação de profissionais: "Farmacêutico e Médico" visando sempre a saúde e o bem-estar do indivíduo (OLIVEIRA et al., 2013).

Portanto, este trabalho analisou a comercialização de medicamentos e produtos para emagrecer em uma drogaria situada no município de Ceres-GO, verificando o gênero do paciente que mais comprou medicamentos para emagrecer, se o medicamento ou produto farmacêutico apresentava prescrição médica para comercialização ou era isento de prescrição, o medicamento ou produto 
farmacêutico que foi mais dispensado para tal finalidade, a especialidade médica que mais emitiu receituários e se os farmacêuticos ou balconistas orientaram os pacientes frente ao tratamento medicamentoso.

\section{MATERIAL E MÉTODOS}

Tratou-se de uma pesquisa de campo observacional de análise quantitativa descritiva de corte transversal. A coleta de dados foi realizada em uma drogaria situada no município de Ceres-GO durante o mês de Agosto no período vespertino nos horários comerciais de 12:00 a 17:00 horas, de segunda-feira à sábado, no ano de 2016. Durante o período de execução da pesquisa e coleta de dados, as pesquisadoras responsáveis pelo estudo estiveram presente no local destinado.

É importante destacar que há mais drogarias na cidade de Ceres-GO, porém a escolha da drogaria para realizar o presente estudo foi devido ao fato que o profissional farmacêutico e o proprietário do estabelecimento de saúde consentiram e autorizaram a realização da pesquisa e a permanência das pesquisadoras no estabelecimento, ao contrário das demais, que não houve o consentimento e autorização.

Os dados foram coletados a partir de um levantamento aplicado em uma planilha de controle, a qual estava presente na drogaria. Nesta planilha continha as seguintes informações para obter os dados: o gênero do paciente que comprou o medicamentos para emagrecer, se o medicamento ou produto farmacêutica para comercialização apresentava prescrição médica, ou seja, se o medicamento tarjado dispensado foi mediante a apresentação receita médica pelo paciente e com retenção da receita, se o medicamento tarjado dispensado foi mediante receita médica, se o medicamento tarjado dispensado foi sem receita e se foi medicamento isento de prescrição (MIP's).

Também verificou quais os produtos (medicamentos sintéticos, fitoterápicos, drogas vegetais, derivados vegetais e outras classes) que foram dispensados para tal finalidade, a especialidade médica (clínico geral, endocrinologista, ginecologista, cirurgião, dermatologista, psiquiatra e entre outras) que emitiu o receituário e por fim se os farmacêuticos ou balconistas orientaram os pacientes sobre o tratamento medicamentoso (posologia e possíveis interações medicamentosas).

Foram incluídos na pesquisa todos os pacientes que apresentaram receitas médicas e adquiriram os medicamentos ou produtos usados para emagrecer e, também todos os pacientes que não continham receitas médicas e compraram os medicamentos ou produtos farmacêuticos e, foram excluídos os pacientes que apresentaram receitas magistrais (medicamentos manipulados). Os dados coletados foram organizados em planilha do programa Excel da Microsoft $\circledast 2013$ e em seguida realizou frequência absoluta e relativa frente aos dados coletados.

\section{RESULTADO E DISCUSSÃO}

Com relação ao gênero do paciente que mais comprou medicamentos e produtos para emagrecer foi o feminino com $75 \%$ (24/32) seguido do gênero masculino com 25\% (08/32) (Tabela 1). Estudo realizado no município de CuiabáMT no ano de 2015 constatou que os clientes do gênero feminino se destacam como usuários deste tipo de medicamento totalizando um percentual de $83,93 \%$ e o gênero masculino apresentando apenas $16,1 \%$ da população que foi analisada, 0 que corrobora com o presente estudo (GONZAGA et al., 2015). 
TABELA 1: Resultados da análise da comercialização de medicamentos e produtos para emagrecer em uma drogaria no município de Ceres-GO, no mês de Agosto de 2016.

\begin{tabular}{|c|c|c|}
\hline & $\begin{array}{l}\text { Frequência } \\
\text { (\%) }\end{array}$ & $\begin{array}{l}\text { Frequência } \\
\text { Absoluta }\end{array}$ \\
\hline \multicolumn{3}{|l|}{ Gênero } \\
\hline Feminino & 75,00 & 24 \\
\hline Masculino & 25,00 & 08 \\
\hline TOTAL & 100 & 32 \\
\hline \multicolumn{3}{|c|}{ Prescrição } \\
\hline $\begin{array}{l}\text { Medicamento tarjado } \\
\text { mediante receituário } \\
\text { retenção da receita }\end{array}$ & 31,25 & 10 \\
\hline $\begin{array}{l}\text { Medicamento tarjado dispensado } \\
\text { mediante receituário médico }\end{array}$ & 06,25 & 02 \\
\hline $\begin{array}{l}\text { Medicamento tarjado dispensado sem } \\
\text { receita médica }\end{array}$ & 15,62 & $05 /$ \\
\hline MIP's & 46,88 & 15 \\
\hline TOTAL & 100 & 32 \\
\hline \multicolumn{3}{|c|}{ Medicamento sintético e fitoterápico } \\
\hline Sibutramina & 71,42 & 10 \\
\hline Sene & 14,29 & 02 \\
\hline Alcachofra & 14,29 & 02 \\
\hline TOTAL & 100 & 14 \\
\hline \multicolumn{3}{|c|}{ Drogas e derivados vegetais } \\
\hline Óleo de coco & 40,00 & 04 \\
\hline Óleo de cártamo & 30,00 & 03 \\
\hline Chá verde & 30,00 & 03 \\
\hline TOTAL & 100 & 10 \\
\hline \multicolumn{3}{|l|}{ Outras Classes } \\
\hline Orlistat & 87,50 & 07 \\
\hline Spirulina & 12,50 & 01 \\
\hline TOTAL & 100 & 08 \\
\hline \multicolumn{3}{|c|}{ Especialidade Médica } \\
\hline Endocrinologista & 66,67 & 08 \\
\hline Clínico Geral & 25,00 & 03 \\
\hline Ginecologista & 08,33 & 01 \\
\hline TOTAL & 100 & 12 \\
\hline \multicolumn{3}{|c|}{ Farmacêutico ou balconista } \\
\hline $\begin{array}{l}\text { Com orientação do tratamento } \\
\text { medicamentoso ao paciente. }\end{array}$ & 31,25 & 10 \\
\hline $\begin{array}{l}\text { Sem orientação do tratamento } \\
\text { medicamentoso ao paciente. }\end{array}$ & 68,75 & 22 \\
\hline TOTAL & 100 & 32 \\
\hline
\end{tabular}


Segundo SOARES et al. (2011), as mulheres são sempre mais susceptíveis a esses tratamentos, pois apesar de apresentarem o peso ideal, estão sempre insatisfeitas com o corpo. Também, devido a pressão exercida pela mídia, as mulheres procuram alternativas que se adéquem ao modelo proposto, neste caso, os medicamentos utilizados para emagrecer.

Já a forma de dispensação dos medicamentos ou produtos farmacêuticos para emagrecer prevaleceu a dispensação dos MIP's com 46,88\% (15/32), seguido da comercialização de $31,25 \%$ (10/32) de medicamentos tarjados dispensados mediante receituário médico e retenção da receita, $15,62 \%(05 / 32)$ medicamentos tarjados dispensados sem o receituário médico e por fim, com 6,65\% (02/32) medicamento tarjado dispensado mediante receita médica, sem precisar de restrição da receita (Tabela 1 ).

Sugere-se que a prevalência dos MIP's é devido ao mesmos não precisam de receitas médicas, facilitando assim, o acesso do consumidor. Outro fator que influência na venda dos MIP'S é a mídia que exibe em suas propagandas atrizes famosas e magras (SANTOS \& GROSSI, 2008; MELO \& OLIVEIRA, 2011). Portanto, a publicidade incentiva a compra dos MIP's e as pessoas acabam se automedicando $\mathrm{e}$, isso é um verdadeiro problema, porque o paciente sem o conhecimento do medicamento pode o consumir de forma errada provocando danos ao organismo (PINA et al., 2012). Outro fator é a indicação de terceiros (vizinhos, amigos e parentes), que já usaram ou "ouviram falar que funciona" o medicamento (MELO \& OLIVEIRA, 2011).

De acordo com MELO \& OLIVEIRA (2011) a automedicação é um fator de destaque das mulheres quanto a insatisfação corporal. Segundo DUTRA et al. (2015) o uso indiscriminado de medicamento sem orientação de um profissional da saúde pode acarretar diversos resultados adversos e indesejados ao usuário. Dentre esses efeitos está o mascaramento de graves sintomas que pertencem à algumas doenças, sendo possível, em alguns casos até mesmo o agravamento do caso clínico apresentado. Os autores ainda ressaltam que o uso indiscriminado de medicamentos pode acarretar intoxicação.

Foi observado no presente estudo que todas sibutraminas foram dispensadas mediante receituário médico e retenção da receita com 31,25\% (10/32). Segundo BRASIL (2014) a prescrição, dispensação e o aviamento de medicamentos que contenham sibutramina deverão ser realizados por meio da Notificação de Receita "B2" e com retenção do receituário médico no estabelecimento de saúde.

No estudo de CRUZ \& SANTOS (2013) realizado no município de Ceres informaram que dos 60 pacientes que adquiriram anfetamínicos, 59\% apresentaram receituário especial entregue pelo médico, $20 \%$ adquiriram sem receita, $13 \%$ pagaram pela consulta só para obter o receituário especial e os outros $5 \%$ utilizaram receituário de outras pessoas.

Com relação a comercialização de medicamentos sintéticos para emagrecer a sibutramina foi o mais vendido com 71,42\% (10/14) (Tabela 1). Esses dados corroboram com o estudo de SILVA et al., (2012) nas cidades de Anápolis, Abadiânia, Jaraguá, Petrolina e Uruaçu no estado de Goiás, em que também prevaleceu a venda da sibutramina com $63,3 \%$ de aquisição do medicamento.

A sibutramina é um inibidor da recaptação de noradrenalina e da serotonina, assim, auxiliam na perca de peso por suprimir o apetite e aumentar o termogênese. O uso deste medicamento tem como efeitos colaterais mais comuns a constipação, 
a cefaleia, boca seca, insônia e problemas cardiovasculares principalmente arritmia, taquicardia e hipertensão (MARQUES \& BAZOTTE, 2014). Ainda tem o efeito rebote que este medicamento causa, ou seja, o ganho de peso após o uso do mesmo é até maior que a perda obtida durante o período de tratamento (BITTENCOURT, 2010). Desta forma, destaca-se a importância indispensável do farmacêutico para a dispensação destes medicamentos visando uma interação entre o farmacêutico e usuário, prevenindo assim, o uso indiscriminado da medicação. Para uma farmacoterapia racional e a obtenção de resultados favoráveis o farmacêutico deve alertar quanto aos riscos e benefícios dos medicamentos, propondo assim, novos hábitos de vida que inclui exercícios físicos e dieta para a redução de peso corporal (OLIVEIRA et al., 2013).

Destaca-se que para os medicamentos anfetamínicos como a anfrepamona, femproporex e mazindol não tiveram prescrições no presente estudo, devido a que os mesmos foram proibidos pela Anvisa no dia 06 de outubro de 2011 e, em seguida, tiveram sua liberação para comercialização em setembro de 2014. Porém, para que esses medicamentos voltem a ser comercializado, deverão ser registrados novamente pelos fabricantes, pois as indústrias não podem utilizar os mesmos documentos de comercialização de antes, ou seja, esses medicamentos têm que ter novo registro e devem passar por novas análises solicitadas pela Anvisa, confirmando a comprovação de eficácia e segurança. Por enquanto, não houve nenhum registro destes medicamentos na Anvisa e, por esse motivo a anfepramona, femproporex e mazindol não estão disponíveis nas drogarias e farmácias de manipulação (BRASIL, 2014).

Com relação à dispensação de fitoterápicos, 14,29\% (02/14) foram de sene e $14,29 \%(02 / 14)$ de alcachofra, os quais são considerados MIP's (Tabela 1). A alcachofra atua no intestino contribuindo com a perda de peso, pois auxilia na digestão das gorduras (BRITO et al., 2014).

Já o sene, segundo o estudo de MANSO (2013), verificou que das 330 pessoas que participaram da pesquisa $142(41,8 \%)$ recorrem ao uso do sene para obter efeito laxativo. No trabalho de CARNEIRO et al., (2008) na cidade de Belo Horizonte- MG, o sene se destacou em terceiro lugar como uma substância de associação com os anorexígenos mais utilizada, com $30,4 \%$ das associações. De acordo TOMÉ \& LOPES (2014), ainda existe uma crença que os fitoterápicos não oferecem perigo a saúde, porém o seu uso inadequado pode induzir problemas graves e até mesmo interações medicamentosas.

Com relação à pesquisa de derivados vegetais e drogas vegetais, $40 \%$ (04/10) das dispensações foram de óleo de coco (Tabela 1). No estudo feito por BERNARDES \& BORGES (2014) apontam que dentre as 14 farmácias analisadas no município de Itumbiara-GO todas houveram solicitações deste derivado vegetal, sendo as mulheres com maior percentual de procura e sempre para fins de emagrecimento, o que corrobora com o presente trabalho. Segundo SILVA (2013) o óleo de coco foi um exemplo de influência da mídia que quando foi apresentado ao público tornou-se uma grande promessa no processo de emagrecimento. No entanto, pesquisas científicas criteriosas apontam que na verdade o óleo de coco funciona como um laxativo, e por ser um óleo pode elevar os níveis sanguíneos de LDL e HDL.

Já a dispensação do óleo de cártamo foi de 30\% (03/10) (Tabela 1). Dentre os principais componentes do óleo de cártamo está o ácido linoléico conjugado (CLA), 
o qual promove a perca da gordura corporal, sendo assim, esta perda pode ser maior se for combinada com a prática de exercícios físicos diários (COSTA et al., 2015).

As drogas vegetais também são solicitadas nas drogarias para fins de perca de peso e suplementação. No presente estudo, o chá verde teve $30 \%(03 / 10)$ da comercialização (Tabela 1). Trabalho realizado por CONDE et al., (2015) verificaram que cerca de $85 \%$ dos atletas de duas academias de Juiz de Fora- MG utilizam a fitoterapia para fins estéticos, sendo o chá verde em primeiro lugar com $61 \%$ o mais consumido por esses praticantes de atividade física, tendo como a finalidade principal o processo de emagrecimento. Com a obtenção da perda de peso, o chá verde apresenta efeitos benéficos para o tratamento e a prevenção de doenças relacionadas com a obesidade, como doenças cardiovasculares, diabetes, câncer e entre outras (FARIA et al., 2015).

Com relação às outras classes medicamentosas, destaca-se o orlistat, o qual teve $87,50 \%(07 / 08)$ da comercialização, seguido da spirulina com $12,50 \%(01 / 08)$ (Tabela 1). De acordo com DUALIB et al. (2008) na classificação das drogas mais utilizadas para o tratamento da obesidade o orlistat ocupa $05^{\circ}$ lugar, sendo seus antecedentes os anorexígenos.

O orlistat é um inibidor reversível da lípase pancreática favorecendo a perda de peso ao reduzir a absorção de gordura alimentar. Depois da dose padrão de 120 $\mathrm{mg}$, três vezes por dia, antes das refeições, o orlistat inibe aproximadamente $30 \%$ da absorção de gordura. Os efeitos adversos mais comuns são os gastrointestinais e incluem incontinência, diarreia, esteatorreia, flatulência, distensão abdominal e dispepsia e até interferência na absorção de algumas vitaminas lipossolúveis, portanto esses efeitos indesejáveis podem ser evitados com diminuição do teor de gordura na dieta (MARQUEZ \& BAZOTTE, 2014).

Já a spirulina é uma bactéria pertencente ao grupo Cyanobacterium conhecida popularmente como grupo das algas verde-azuladas. O consumo da spirulina confere uma sensação de saciedade que inibe a fome, isso é devido que tem a capacidade de expandir (entumecer) ao contato com a água do trato gastrointestinal (FRANÇA et al., 2008; SILVA, 2013).

Com relação à especialidade médica, foi constatado a predominância do endocrinologista com $66,67 \%(08 / 12)$ que mais emitiu receituário médico para a dispensação de medicamentos e produtos para emagrecer, seguido do clínico geral com 25\% (03/12) e em terceiro o ginecologista com 8,33\% (01/12). Esses dados corroboram com o estudo de PEREIRA et al. (2012) realizado na cidade de Curitiba$\mathrm{PR}$, em que o maior número de receitas para emagrecer está relacionado com o profissional endocrinologista, sendo $70 \%$ responsável pela maioria das prescrições.

Também o trabalho desenvolvido em uma drogaria localizada na região do Distrito Federal foi observado que as prescrições de medicamentos anorexígenos foram realizadas com $43,75 \%$ por endocrinologistas, $37,5 \%$ por clínicos médicos e $31,3 \%$ por médicos de outras especialidades (ginecologistas, gastroenterologistas e dermatologistas) (OLIVEIRA \& VASCONCELOS, 2012). Em contrapartida, a análise dos receituários de controle especial contendo medicamentos anorexígenos realizado na farmácia de manipulação no município de Pelotas- $\mathrm{RS}$ constatou a prevalência de médicos da especialidade Clínica Geral com 100\% (169/169), sendo os que mais prescreveram em ambos os períodos avaliados devido ao acesso mais rápido dos pacientes a estes médicos (LAILA et al., 2013). 
Quando da orientação do farmacêutico ou balconista ao paciente frente ao tratamento medicamentoso, apenas $31,25 \%$ (10/32) dos pacientes tiveram orientação farmacêutica e cerca de $68,75 \%$ (22/32) não tiveram nenhum tipo de orientação sobre modo de usar e possíveis riscos dos medicamentos.

Segundo estudo de SILVA et al. (2012) que avaliaram o consumo de anorexígenos nas cidades de Goiás como Anápolis, Abadiânia, Jaraguá, Petrolina e Uruaçu observaram que das 85 pessoas entrevistadas apenas $31,7 \%$ declararam terem sido orientados por um profissional farmacêutico.

É imprescindível a participação do Farmacêutico exercendo a atenção farmacêutica, a fim de evitar, por exemplo, possíveis erros de prescrição e dispensação. Assim, o Farmacêutico contribui para 0 uso racional dos medicamentos, trabalha em prol da promoção da saúde e o paciente terá consciência dos riscos do uso abusivo dos medicamentos (MAGALHÃES et al., 2016).

De acordo com o supracitado, apesar da sibutramina realmente ser eficaz no tratamento da obesidade, deve-se ter cautela ao utilizar o medicamento para evitar os efeitos adversos. Diante disso, faz-se necessária a orientação sobre seu uso por um médico ou farmacêutico (MARINI et al., 2014), o que não foi observado no presente estudo.

\section{CONCLUSÃO}

O gênero que mais comprou medicamento para emagrecer foi o feminino, predominou a dispensação de MIP's, a sibutramina foi o medicamento mais comercializado mediante retenção de receita, o óleo de coco foi o derivado vegetal mais vendido durante o período da pesquisa, em relação a outras a classes de produtos farmacêuticos o orlistat se destacou como o medicamento tarjado dispensado sem receituário médico, grande parte dos pacientes buscam ajuda do profissional endocrinologista para indicação de tais medicamentos e produtos para emagrecer e por fim, a maioria dos pacientes não receberam orientação farmacêutica em relação ao tratamento medicamentoso e possíveis efeitos adversos.

\section{REFERÊNCIAS}

ABESO. Atualização das Diretrizes para o Tratamento Farmacológico da Obesidade e do Sobrepeso. Edição Especial, p. 76, 2010. Disponível em: <http://www.abeso.org.br/pdf/diretrizes2010.pdf>. Acesso em: 25 maio 2016

ABIFARMA. Automedicação e uso incorreto de medicamentos podem levar à morte. 2016. Disponível em: <http://www.guiadafarmacia.com.br/noticias/saude/9502-automedicacao-euso-incorreto-de-medicamentos-podem-levar-a-morte>. Acesso em: 24 de maio de 2016.

BERNARDES, Q. C. B; BORGES, C.C. O crescente uso de medicamentos e produtos emagrecedores: bases cientificas $x$ dados empíricos. IN: Anais. Anais da Semana Interdisciplinar, Seminário de Iniciação Científica e Semana da Família do Câmpus de Itumbiara, v. 1 n. 1, 2014.

BITTENCOURT, P. C. T. Uso Abusivo de Medicamentos. Revista Visão Acadêmica, v. 9, n.2, p. 1-22, 2010. 
BRASIL. Agência Nacional de Vigilância Sanitária (ANVISA). Resolução RDC n 50, de 25 de setembro de 2014. Dispõe sobre as medidas de controle de comercialização, prescrição e dispensação de medicamentos que contenham as substâncias anfepramona, femproporex, mazindol e sibutramina, seus sais e isômeros, bem como intermediários e dá outras providências. 2014. Disponível em: <http://portal.anvisa.gov.br/documents/33880/2568070/reprdc0050_25_09_2014.pdf/ d04dec76-4dbb-4d04-a721-50bd191a1a9b?version=1.0>. Acesso em: 27 de nov. de 2016.

Agência Nacional de Vigilância Sanitária (ANVISA). Avaliação de Eficácia e Segurança dos medicamentos Inibidores do Apetite. 2011 a. Disponível em: $<$ http://www.anvisa.gov.br/hotsite/anorexigenos/pdf/Nota Tecnica Anorexigenos.pdf $>$.

Acesso em: 24 de maio 2016.

Agência Nacional de Vigilância Sanitária (ANVISA). Riscos X Benefícios. Especialistas discutem perfil de segurança da Sibutramina. $2011 \mathrm{~b}$. Disponível

em:<http://portal.anvisa.gov.br/wps/content/anvisa+portal/anvisa/sala+de+i mprensa/menu+noticias+anos/2011+noticias/riscos+X+beneficios+especiali stas+discutem+perfil+de+seguranca+da+sibutramina $>$. Acesso em: 25 de maio de 2016.

BRITO, A. G. R.; FREITAS, C. L.; GALVÃO, R. C.; NUNES, J. L. S.; EMILIANO, M. D. S.; SANTOS, R. S. Fitoterapia: uma alternativa terapêutica para o cuidado em Enfermagem-relato de experiência. Biota Amazônia Open Journal System, v. 4 n. 4 p. 15-20, 2014.

CARNEIRO, M. F. G; JUNIOR, A. A. G; ACURCIO, F. A. Prescrição, dispensação e regulação doconsumo de psicotrópicos anorexígenos em Belo Horizonte, Minas Gerais, Brasil. Revista Caderno de Saúde Pública, v. 8 n. 24, p. 1763-72, 2008.

CARVALHO, F. Q. M.; MACIEIRA, P. R. C. Medicina e direito, remédios para emagrecer e Anvisa: a necessidade de uma atuação transdisciplinar para garantir constitucionalidade e legalidade. 2012. Disponível em: <http://www.egov.ufsc.br/portal/conteudo/medicina-e-direito-rem\%C3\%A9dios-paraemagrecer-e-anvisa-necessidade-de-uma atua\%C3\%A7\%C3\%A3o-transdiscipl>. Acesso em: 24 maio 2016.

CONDE, B. E.; MACEDO, A. L.; MARTINS, A. E.; FONSECA, A. S.; SIQUEIRA, A. M.; SOUZA, G. H. L.; ROGÉRIO, I. T. S. Estudo Crítico sobre utilização de fitoterápicos por praticantes de exercícios físicos em academia de musculação. Revista Ciências Biológicas e da saúde. v. 5 n. 16 p. 33-47, 2015.

COSTA, N. C.; FIGUEIREDO, P. M. S.; SILVA, D. P.; CHAGAS JÚNIOR, A. F.; SCHEIDT, G. N. Suplementos alimentícios contendo óleo de coco e cártamo para o tratamento da obesidade: Seus interferentes nos padrões do perfil lipídico. Journal of Bioenergy and Food Science, v. 2, n. 1, 2015. 
CRUZ, A. C. S; SANTOS, E. N. Avaliação do consumo de medicamentos para emagrecer em farmácias, nomunicípio de Ceres - Goiás, Brasil. Revista da Universidade Vale do Rio Verde Três Corações, v. 10 n. 1 p. 402-409, 2013.

DUALIB, P. M. COUTINHO, W. F.; COSTA, C. P.; DIB, S. A. "Obesidade". Revista. Brasileira de Medicina, v. 65 n. 12 p. 26-31, 2008.

DUTRA, J. R; SOUZA, S. M. F; PEIXOTO, M. C. A influência dos padrões de beleza veiculados pela mídia. Como fator decisório na automedicação com moderadores de apetite por mulheres no município de Miracema-RJ. Revista Transformar, n. 7, p. 194-213, 2015.

FARIA, D. P; ESCHER, A; FRANÇA, S.A. Chá verde como coadjuvante no tratamento as obesidades e suas comorbidades. Revista Uniciências. v. 14 n. 2 p. 105-126, 2015.

FRANCA, G. A. M.; GRISI, L. M; SILVA, A. S. Efeito agudo do consumo da espirulina máxima no dano muscular induzido por exercícios em atletas corredores de Endurance. Revista Brasileira de Nutrição Esportiva. v. 2 n. 7 p. 11-20, 2008.

GONZAGA, J. B.; SIQUEIRA, F. S.; ZAMPRONE, J. T.; FRANCO, B.; CONSTANTINO, S. K. C.; SILVA, E. A. J.; OLIVEIRA, R. G. Análise das prescrições de sibutramina dispensadasem drogarias no município de Cuiabá-MT, Brasil. Revista Infarma Ciências Farmacêuticas, v. 27 n. 1, p. 33-37, 2015.

LAGARES, T. Uso indevido causa morte de 20 mil por ano no Brasil. 2015. Disponível em: <http://www.dm.com.br/cotidiano/2015/04/uso-indevidocausa-morte-de-20-mil-por-ano-no-brasil.html>. Acesso em: 24 de maio 2016.

LAILA, H. J. E. A.; MENEZES, A. P. S.; NUNES, M. F. S.; HORTA, R. N. ZAGO, A. C. Análise de prescrições destinadas ao emagrecimento em farmácia magistral antes e após a vigência da RDC $N^{\circ}$ 52. Infarma-Ciências Farmacêuticas, v. 25, n. 4, p. 182-187, 2013.

MAGALHÃES, A. E. C; DINELLY, C. M. N; OLIVEIRA, M. A. S. Psicotrópicos: perfil de prescrições de benzodiazepínicos, antidepressivos e anorexígenos a partir de uma revisão sistemática. Eletronic Journal of Pharmacy, v. 13 n. 3 p. 111-122, 2016.

MANSO, C. I. M. P. Consumo de laxantes particularmente de sene numa farmácia do Nordeste Transmontano. 2013. Disponível em:<https://bibliotecadigital.ipb.pt/bitstream/10198/9211/1/Cristiana\%20Manso.pdf> Acesso em: 21 de novembro de 2016.

MARINI, D. C; SILVA, L. O; OLIVEIRA, D. C. Perfil da dispensação e do uso de sibutramina para tratamento da obesidade. Revista FOCO. n.7 p. 61-78, 2014. 
MARQUES, A. C. R; BAZOTTE, R. B. Efeito da suplementação com ácido linoléico conjugado no tratamento da obesidade: estudo de revisão literária em modelo experimentais animais e em humanos. Revista de Saúde e Biologia, v. 9 n. 3 p. 107-117, 2014.

MARQUES, L. A. M. Atenção Farmacêutica em distúrbios menores. São Paulo: Livraria, Editora Medfarma, 2008.

MARTINS, M. C.; GARLET, T. M. B. Desenvolvendo e divulgando o conhecimento sobre plantas medicinais. Revista Eletrônica em Gestão, Educação e Tecnologia Ambiental, v. 20, n. 01, p. 438-448, 2016.

MELO, C. M.; OLIVEIRA, D. R. O uso de inibidores de apetite por mulheres: um olhar a partir da perspectiva de gênero. Ciência \& Saúde Coletiva, v.16, n. 5, p. 2523-2532, 2011.

OLIVEIRA, D. P. G.; LAGES, L. T. R; ASSIS, J. R. Prevalência do uso de anorexígenos em acadêmicos de uma instituição privada na cidade de Montes Claros, MG. Infarma-Ciências Farmacêuticas, v. 25, n. 1, p. 17-23, 2013.

OLIVEIRA, J. P, VASCONCELOS, R. B. O impacto dos atos regulatórios da agência nacional de vigilância sanitária sobre a dispensação de psicotrópicos anorexígenos. Revista de Divulgação Científica Sena Aires, v. 1 n.2 p. 147-154, 2012.

PEREIRA, F A.; NEVES, E. B.; MASCARENHAS, L. P.; PIETROVSKI, E. F. Avaliação de prescrições de anorexígenos em farmácia magistral. Revista Brasileira de Farmácia, v.93, n. 4, p. 481-486, 2012.

PINA, H.; CALAZANS, J.; GOMES, J. P.; CAVALCANTI, M.; SOUZA, P.; ALVES, R. FREITAS, R. Publicidade de medicamentos e o incentivo à automedicação. UNICAP-Universidade Católica de Pernambuco, p.1-7, 2012.

PRADO, C. N.; NEVES, D. R. J.; SOUZA, H. D.; NAVARRO, F. O uso de fitoterápicos no tratamento da obesidade. Revista Brasileira de Obesidade, Nutrição e Emagrecimento, v. 4, n. 19, 2010.

ROSSE, W. J. D.; MOURO, V. G. S; FRANCO, A. J.; CARVALHO, C. A. Perfil da automedicação em acadêmicos do curso de farmácia da Univiçosa, Viçosa, MG. Revista Brasileira de Farmácia, v.92, n.3, p.186-190, 2011.

SANTOS, A. M.; GROSSI, P. K. Mídia, sociedade de consumo e saúde: quando comprar torna-se um remédio. Boletim da saúde, v. 21, n. 02, p. 62-66, 2008.

SILVA, J. R.; DE OLIVEIRA, E. N. F.; FERREIRA, A. G. Avalição do consumo de anorexígenos derivados de anfetamina em cidades de Goiás-GO. Ensaios e Ciência: C. Biológicas, Agrárias e da Saúde, v. 16, n. 3, 2012. 
SILVA, M. E. M. Estudo de plantas medicinais utilizadas popularmente no tratamento da obesidade em Araranguá. 2013. Disponível em: < https://ead.ufsc.br/biologia/files/2014/05/Maria-Eduarda-Moreira-da-Silva.pdf> Acesso em: 18 de outubro de 2016.

SOARES, V. C. G.; VECHIATO, C.; PIERINI, E. C.; DEMARCHI, G. M.; FRANCESCONI, E. P. M.S.; OLIVEIRA, D. A. G. Autoimagem corporal associada ao uso de sibutramina. Revista do instituto de ciência da saúde, v. 29 n. 1 p. 45-51, 2011.

TOMÉ, A; LOPES, S. S. Comércio de Alimentos de Apresentação Não Convencional, Drogas Vegetais e Fitoterápicos: Oferta em Drogarias de Araucária, PR. Revista Brasileira de Terapia e Saúde, v. 4 n. 2 p. 7-14, 2014.

WEISHEIMER, N.; COSTA FILHO, P. F.; NEVES, R. P. C.; SOUSA, R. M.; PINTO, D. S.; LEMOS, V. M. Fitoterapia como alternativa terapêutica no combate a obesidade. Revista ciência e saúde Nova Esperança, v. 13, n. 01, p. 103$111,2015$. 